\title{
Comparison of static-feedforward and dynamic-feedback neural networks for rainfall-runoff modeling
}

\author{
Yen-Ming Chiang, Li-Chiu Chang, Fi-John Chang* \\ Department of Bioenvironmental Systems Engineering and Hydrotech Research Institute, \\ National Taiwan University, Taipei 10770, Taiwan, ROC
}

Received 24 April 2003; revised 14 November 2003; accepted 10 December 2003

\begin{abstract}
A systematic comparison of two basic types of neural network, static and dynamic, is presented in this study. Two backpropagation (BP) learning optimization algorithms, the standard BP and conjugate gradient (CG) method, are used for the static network, and the real-time recurrent learning (RTRL) algorithm is used for the dynamic-feedback network. Twenty-three storm-events, about 1632 rainfall and runoff data sets, of the Lan-Yang River in Taiwan are used to demonstrate the efficiency and practicability of the neural networks for one hour ahead streamflow forecasting. In a comparison of searching algorithms for a static network, the results show that the CG method is superior to the standard BP method in terms of the efficiency and effectiveness of the constructed network's performance. For a comparison of the static neural network using the CG algorithm with the dynamic neural network using RTRL, the results show that (1) the static-feedforward neural network could produce satisfactory results only when there is a sufficient and adequate training data set, (2) the dynamic neural network generally could produce better and more stable flow forecasting than the static network, and (3) the RTRL algorithm helps to continually update the dynamic network for learning - this feature is especially important for the extraordinary time-varying characteristics of rainfall-runoff processes.

(c) 2004 Elsevier B.V. All rights reserved.
\end{abstract}

Keywords: Rainfall-runoff processes; Streamflow forecasting; Neural networks; Static systems; Dynamic systems

\section{Introduction}

A rainfall-runoff model is one of the most prominent techniques for real-time flood forecasting in Taiwan, with its high mountains and steep slopes all over the island. Typhoons (around four times a year) and thunderstorms bring heavy rainfalls that can flood downstream cities within a few hours. For decades,

\footnotetext{
* Corresponding author.

E-mail address: changfj@ntu.edu.tw (F.-J. Chang).
}

we have not coped well with floods owing to the complex watershed rainfall-runoff process that is highly non-linear and dynamic in nature. Flood forecasting remains one of the most challenging and important tasks of operational hydrology.

In the past, a considerable research has been carried out in developing rainfall-runoff processes based on deterministic/conceptual models (Hydrological Engineering Center, 1990) or stochastic models (Salas et al., 1985). The application of conceptual models offers the possibility of identifying processes 
or improving our knowledge in a specific watershed. However, in nature, different processes interact across different scales in a non-linear way, and such interactions are poorly understood and are not well represented (Beven, 2001). Moreover, the need for a great amount of field experimental data to model the underlying processes and/or sophisticated optimizing techniques to calibrate the models has limited their application. Clearly, there is a strong need to explore alternative approaches to develop better models.

The Artificial Neural Network (ANN) is a relatively new computational tool that is inspired by neurobiology to perform brain-like computations. The attractiveness of ANNs comes from information processing characteristics such as non-linearity, parallelism, noise tolerance, and learning and generalization capability. Due to its immense ability for modeling complex nonlinear systems, the application of ANNs to various aspects of hydrological modeling has undergone much investigation in recent years and has provided many promising and exciting results in the field of hydrology (Karunanithi et al., 1994; Thirumalaiah and Deo, 1998; Dawson and Wilby, 1998; Campolo et al., 1999; Sajikumar and Thandaveswara, 1999; Tokar and Johnson, 1999; Zealand et al., 1999; Chang et al., 2001, 2002; Chang and Chen, 2001; Cameron et al., 2002; Sivakumar et al., 2002), water resources (Smith and Eli, 1995; Shamseldin, 1997; Golob et al., 1998; Coulibaly et al., 2001; Chang and Chang, 2001; Dawson and Wilby, 2001; Elshorbagy et al., 2000) and rainfall forecasting (French et al., 1992; Luk et al., 2000). A comprehensive review of the application of ANNs to hydrology can be found in the ASCE Task Committee report $(2000 \mathrm{a}, \mathrm{b})$ and in Maier and Dandy (2000), and also in a specialized publication (Govindaraju and Ruo, 2000).

After McCulloch and Pitts (1943) established the first simple neural network, many different types of ANN have been proposed. The most widely used structures are the back-propagation (BP) neural network (Rumelhart et al., 1986), the self-organizing feature mapping (SOM) network (Kohonen, 1982), the Hopfield neural network (Hopfield, 1982), the radial basis function (RBF) neural network (Powell, 1985) and the recurrent neural network (Williams and Zipser, 1989). All these models have been used and reported with satisfactory results in the field of hydrology, especially in streamflow forecasting. For example, Hsu et al. (1995) used BP to provide a better representation of the rainfall-runoff relationship than the ARMAX (autoregressive moving average with exogenous inputs) time-series approach or the conceptual SAC-SMA (Sacramento soil moisture accounting) model; Abrahart and See (2000) provided improved performance by applying SOM classification of different event types; Chang and Chen (2003) demonstrated the RBF network to be a reliable way for water-stage forecasting in an estuary; and Chang et al. (2002) showed that the real-time recurrent learning (RTRL) algorithm can be applied with high accuracy for real-time streamflow forecasting.

Since, there are so many types of neural network, we are frequently faced with questions such as which neural network is the best fit or which neural network should be used for a specific problem. Unfortunately, there is no general methodology or guideline to answer these kinds of questions because we are still in the mid-stage of exploring the capability of using ANNs for modeling hydrological processes. To deal with this problem, one still needs to investigate existing neural networks and to assess their applicability and effectiveness based on the available inputoutput patterns and the qualitative and quantitative aspects of the data sets. In this study, we aim to provide a systematic comparison of two basic types of neural network-static-feedforward and dynamicfeedback algorithms - in modeling rainfall-runoff processes.

\section{Feedforward and feedback neural networks}

There are many different taxonomies for describing ANNs, such as learning/training paradigms, network topology, and network function. Popular examples of classifying ANNs can be found in Haykin (1999) and Ham and Kostanic (2001). ANN applications require an assessment of neural network architectures. The assessment might include: (1) selecting a common pre-existing structure for which training algorithms are available; (2) adapting a preexisting structure to suit a specific application. In viewing network topologies and structures of the ANNs used in the field of hydrology, we can distinguish them into two different generic neural 
network types: feedforward and feedback (recurrent) networks. The architectures and learning algorithms of these networks are briefly described in the sections that follow.

\subsection{Feedforward architecture}

The topology of feedforward ANNs consists of a set of neurons connected by links in a number of layers. The basic configuration usually consists of an input layer, a hidden layer and an output layer. A multilayer feedforward ANN is shown in Fig. 1. For operation of the feedforward ANN, the available data are usually divided into three subsets.

1. Training phase. Network learning (i.e. weight adjustment) by using the whole training data set once is called a learning cycle or one epoch. During the training phase, the training data set is presented repeatedly to the network until the weight values are determined.

2. Validation phase. The most important task in the validation phase is to check the performance of the network to determine the epoch at which training should be stopped to avoid over-fitting or overtraining. If the validation data set indicates that the network is overtrained, then the network should be retrained using a different number of neurons and/or parameter values.

3. Testing phase. The testing data set is used for final evaluation of the network performance. The output of the network is produced solely by input-output mapping during the testing phase.

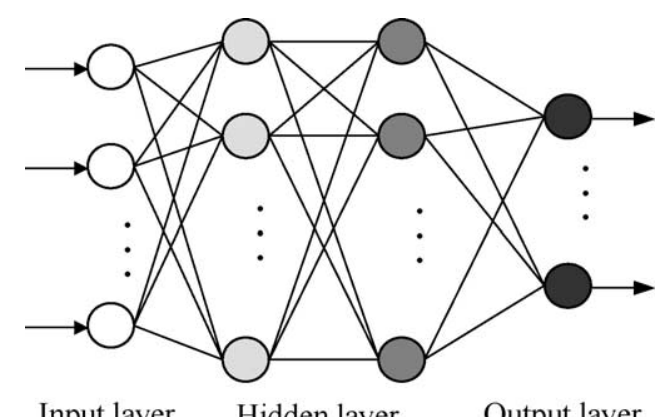

Fig. 1. The architecture of a multilayer feedforward neural network.
It appears the feedforward networks implanted fixed-weight mapping from the input space to the output space. The weights of a feedforward network are fixed after training, so the state of any neuron is solely determined by the input-output pattern and not the initial and past states of the neuron, that is, there is no dynamics involved. Consequently, this type of feedforward architecture is classified as a static neural network. The advantage of the staticfeedforward neural network is that the network can easily be built with a simple optimizing algorithm (e.g. Steepest descent method) and is the most popular neural network architecture in use today. Nonetheless, the static-feedforward neural network also has several drawbacks for some applications. First, it may fail to produce a satisfactory solution because the training data are insufficient in size. Second, the static-feedforward neural network cannot cope well with major changes that were never learned in the training phase. Finally, the staticfeedforward neural network easily falls into a local minimum and the speed of convergence of the network can be very slow when the number of input in the data set is large.

\subsection{Feedback architecture}

There are several types of feedback architecture, which has feedback connections from the output layer to the input layer or from the hidden layer to the input layer. In other words, a feedback architecture distinguishes itself from a feedforward architecture, in that it has at least one feedback link. A recurrent neural network is shown in Fig. 2, which has feedback

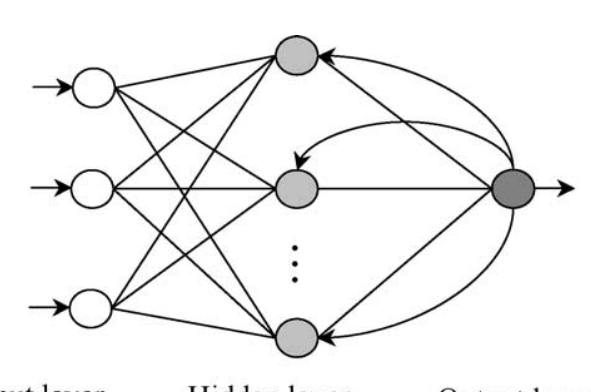

Input layer Hidden layer Output layer

Fig. 2. The architecture of a recurrent neural network. 
connections originating from the outputs of the output layer neurons to its hidden layer. Since, the neurons have one or more feedback link whose state varies with time, the feedback architecture is also called a dynamic neural network. The presence of a feedback link has a profound impact on the learning capability of the network and on its performance. Because the feedback neural networks have adjustable weights, the state of its neuron depends not only on the current input signal, but also on the previous states of the neuron. The advantage of the dynamic-feedback neural network is that it can effectively decrease the network's input dimension and therefore the training time. However, due to the nonlinear nature of unit activation output characteristics and the weight adjustment strategies, the network stability is often difficult to ascertain.

\subsection{Learning algorithm}

There are various types of algorithms for training the network Basically, the purpose of every algorithm is to estimate the local error at each neuron and systematically update the network weights. In this study, the static-feedforward neural network was trained with two different algorithms - the standard BP algorithm and the conjugate gradient (CG) algorithm to estimate/assess their search efficiency and accuracy in this application. The dynamic-feedback neural network was trained with a RTRL algorithm. The details of the above algorithms are given in the following paragraph.

\subsubsection{Standard back-propagation algorithm}

The BP network is trained using supervised learning, so the goal of this algorithm is to decrease global error. To obtain the optimal values of the connected weights such that the energy function is a minimum, the standard BP algorithm searches the error surface by using the steepest descent method. The connected weights are adjusted by moving a small step in the direction of the negative gradient of the energy function at each iteration.

The standard BP algorithm for a static neural network works as follows.
Define the instantaneous overall network error at time $t$ as

$E(t)=\frac{1}{2} \sum_{j=1}^{J}\left[T_{j}(t)-z_{j}(t)\right]^{2}$

where $T_{j}(t)$ is the target value of neuron $j$ at time $t$; and $z_{j}(t)$ is the network output of neuron $j$ at time $t$.

The energy function is obtained by summing $E(t)$ over all time $T$.

$E_{\mathrm{total}}=\sum_{t=1}^{T} E(t)$

The weight change for any particular weight $W_{j i}$, which connects neuron $j$ in this layer with neuron $i$ in the previous layer, can thus be written as

$\Delta W_{j i}=-\eta \frac{\partial E}{\partial W_{j i}}$

where $\eta$ is the learning rate parameter.

Using the chain rule for partial derivatives, the weight change can be generalized as

$\Delta w_{j i}^{n}=\eta^{n} \delta_{j}^{n} z_{j}^{n-1}$

where $z_{j}^{n-1}$ represents the output value of neuron $j$ in the $(n-1)$ th layer, $\delta_{j}^{n}=\left(T_{j}-z_{j}^{n}\right) g\left(v_{j}^{n}\right)$ for the output layer, $\delta_{j}^{n}=\left(\sum_{h=1} \delta_{h}^{n+1} w_{h j}^{n+1}\right) g\left(v_{j}^{n}\right)$ for the hidden layers, and $g(\cdot)$ represents the first derivative of nonlinear activation function.

Further details on the standard BP algorithm can be found in the literature (Rumelhart et al., 1986).

\subsubsection{Conjugate gradient algorithm}

The CG method is a well-known numerical technique used for solving various optimization problems. This technique differs from the standard BP algorithm in updating directions of weights. In other words, the CG algorithm searches a system of conjugate directions on the error surface and updates the weights along these directions. In practice, the process makes good uniform progress toward the solution at every time step and has been found to be effective in finding better optimization than the standard BP algorithm. The CG algorithm is well summarized by Ham and Kostanic, 2001. 


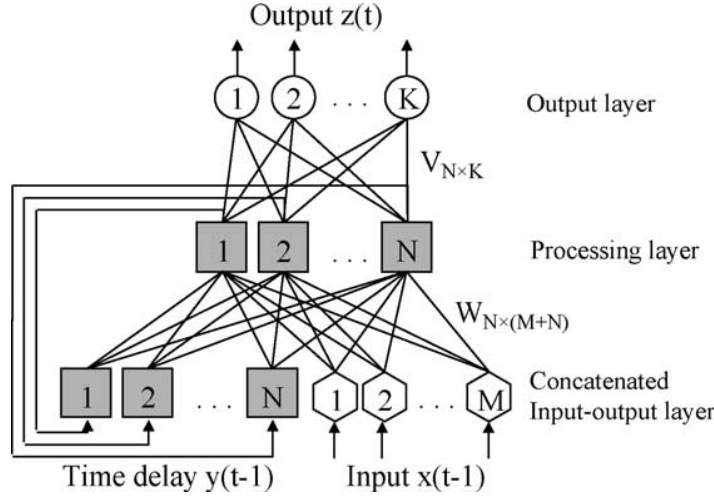

Fig. 3. The architecture of a RTRL network.

\subsubsection{Real-time recurrent learning (RTRL) algorithm}

Consider a three-layer fully interconnected recurrent neural network as shown in Fig. 3, which includes $M$ external inputs, $N$ hidden neurons and $K$ outputs. Let $V$ and $W$ denote the $N \times K$ weight matrix and $N(M+N)$ recurrent weight matrix, respectively. The net activity of neuron $j$ at time $t+1$, for $j \in B$, is computed by

$\operatorname{net}_{j}(t)=\sum w_{j i}(t-i) u_{i}(t-1)$

The output of neuron $j$ in the processing layer is given by passing $\operatorname{net}_{j}(t)$ through the nonlinear transfer function $f(\cdot)$, yielding

$y_{j}(t)=f\left(\operatorname{net}_{j}(t)\right)$

The net output of neuron $k$ in the output layer at time $t$ is computed by

$\operatorname{net}_{k}(t)=\sum k_{k j}(t) y_{j}(t)$

$z_{k}(t)=f\left(\operatorname{net}_{k}(t)\right)$

To minimize the energy function, the steepest descent method is applied to adjust the weights ( $V$ and $W$ ) along the negative of $\nabla E_{\text {total }}$. The weight change for any particular weight $v_{k j}(t)$ can thus be written as

$\Delta v_{k j}(t)=-\eta_{1} \frac{\partial E(t)}{\partial v_{k j}(t)}$

By using the chain rule, the partial derivative of $v_{k j}(t)$ can be obtained as follows

$\frac{\partial E(t)}{\partial v_{k j}(t)}=\sum_{k=1}^{K}-\mathrm{e}_{k}(t) f^{\prime}\left(\operatorname{net}_{k}(t)\right) y_{j}(t)$
Therefore,

$\Delta v_{k j}(t)=\eta_{1} \sum_{k=1}^{K} \mathrm{e}_{k}(t) f^{\prime}\left(\operatorname{net}_{k}(t) y_{j}(t)\right.$

The same method is also implemented for weight $w_{m n}(t-1)$. Then, the weight changes can be computed as

$\Delta w_{m n}(t-1)=\eta_{2}\left[\sum_{k=1}^{K} \mathrm{e}_{k}(t) f^{\prime}\left(\operatorname{net}_{k}(t)\right) v_{k j}(t)\right] \pi_{m n}^{j}(t)$

where

$$
\begin{aligned}
\pi_{m n}^{j}(t)= & f^{\prime}\left(\operatorname{net}_{j}(t)\right) \\
& \times\left[\sum_{i \in B} w_{j i}(t-1) \pi_{m n}^{i}(t-1)+\delta_{m j} u_{n}(t-1)\right]
\end{aligned}
$$

$\eta_{1}$ and $\eta_{2}$ are the learning-rate parameter.

Detailed information on the RTRL algorithm can be found in Williams and Zipser (1989) and Chang et al. (2002).

\section{Application}

This section describes the area of study and provides an overview of how to determine the static-feedforward neural network structure. The above methodologies are applied to the Lan-Yang River for streamflow forecasting. The standard BP and CG algorithms are compared with different input dimensions and hidden neurons to find the best staticfeedforward neural network structure.

\subsection{Description of study area}

The Lan-Yang River is located in northeastern Taiwan with a drainage area of approximately $978 \mathrm{~km}^{2}$. The Lan-Yang River is about $73 \mathrm{~km}$ in length and the average channel slope is $1 / 55$. Locations of the study area and gauge stations used are shown in Fig. 4, where the rain-gauge and streamflow stations are denoted by circles and by triangles, respectively. Hourly streamflow measurements were available from the gauging station located at Lan-Yang Bridge. The hourly data were collected from these gauge stations during the period from 1981 


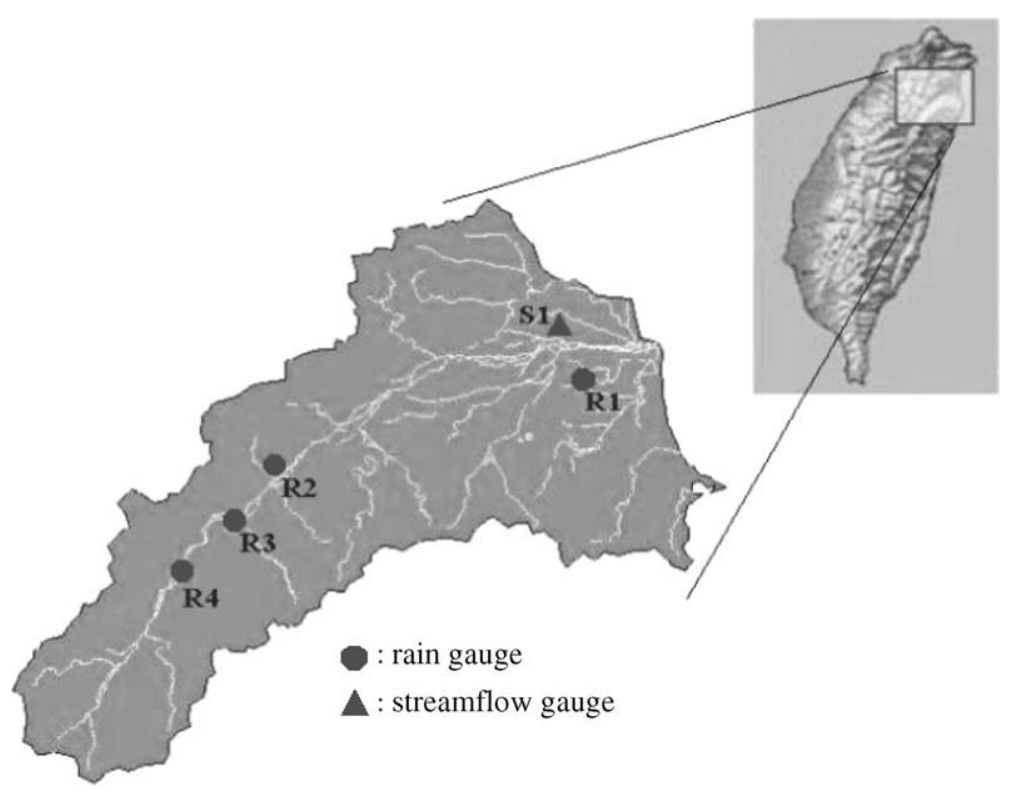

Fig. 4. Location of study area and gauge stations.

to 2000, consisting of 23 storm events with 1632 observations. These events are arbitrarily divided into three different subsets, namely training, validation, and testing subsets. In order to investigate the characteristics and compare the performance between the static-feedforward neural network and dynamicfeedback neural network, we designed four different cases with different training data length and contents. Table 1 shows the diverse length and peak flow of the training data set of the four cases. Cases 1 and 2 have more training data than cases 3 and 4 . Cases 1 and 3 include a peak flow whose value is close to the peak flow in the testing data set, while the peak flow in cases 2 and 4 is much lower than the peak flow in the testing data set.

\subsection{Determining an ANN structure}

After a particular ANN architecture and an appropriate training algorithm are selected, the next step is to determine the network structure The learning ability and performance of an ANN depends on a suitable structure. Generally speaking, the network structure is usually decided on the input dimension, the number of hidden layers, the number of hidden neurons and the output dimension.

\subsubsection{Selection of the number of hidden layers}

There are a number of studies indicating that one or two hidden layers would generally have better convergence, because more than two hidden layers would result in the ability of convergence to reduce gradually and produce many local minima. For instance, Abrahart and See (2000) indicate that the use of two or more hidden layers might not substantially improve the network performance but only add to the training time; and Minns and Hall (1996) state that an alternative approach might be to increase the number of neurons in the hidden layer rather than to add another layer to the network and a single hidden layer should be sufficient for

Table 1

Different length and peak flow of training data set

\begin{tabular}{|c|c|c|c|c|c|c|}
\hline & \multicolumn{2}{|c|}{ Training set } & \multicolumn{2}{|c|}{ Validation set } & \multicolumn{2}{|c|}{ Testing set } \\
\hline & Events & $Q_{\mathrm{p}}$ & Events & $Q_{\mathrm{p}}$ & Events & $Q_{\mathrm{p}}$ \\
\hline Case 1 & 15 & 3530 & 5 & 3350 & 3 & 3640 \\
\hline Case 2 & 15 & 3090 & 5 & 3350 & 3 & 3640 \\
\hline Case 3 & 6 & 3530 & 6 & 3020 & 11 & 3640 \\
\hline Case 4 & 6 & 2916 & 6 & 3020 & 11 & 3640 \\
\hline
\end{tabular}

Where $Q_{\mathrm{p}}$ is observed peak flow (cms). 

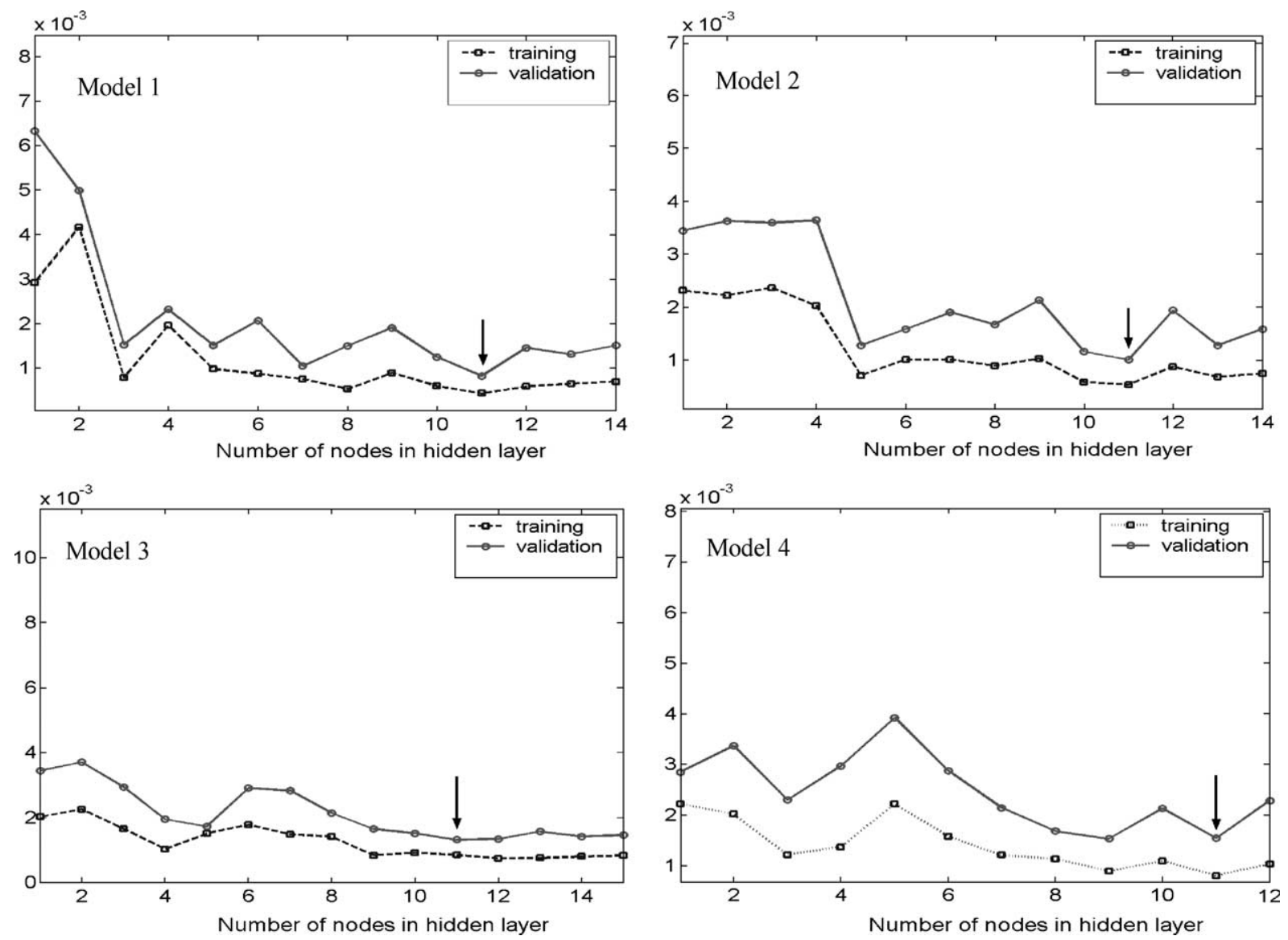

Fig. 5. The selection of hidden neurons from models 1 to 4 for standard BP.

the majority of real-world applications. Therefore, a single hidden layer was adopted in this work.

\subsubsection{Selection of input and output dimension}

While static-feedforward neural networks are very popular, they lack feedback connections to effectively remember and handle the previous states of information. One way that information can be introduced in static-feedforward neural networks is to input a time delay pattern that constitutes the tapped delay line information. Therefore, this network must determine the input variables, output variables and the lag time of the basin before constructing rainfall-runoff procedures in order to increase its accuracy. In general, the selection of input variables and output variables is problem dependent. The appropriate input variables will allow the network to successfully map to the desired output and avoid loss of important information. In the present study, the input dimensions are determined by the input variables and the lag time. To determine an appropriate static-feedforward neural network structure for forecasting the streamflow at time $t+1$ in this selected basin, we develop four different models for the standard BP and CG algorithms, namely:

Model 1: $Q(t+1)=f\left(Q(t), R_{1}(t), R_{2}(t), R_{3}(t)\right.$, $\left.R_{4}(t)\right)$

Model 2: $\quad Q(t+1)=f\left(Q(t), \quad Q(t-1), \quad R_{1}(t)\right.$, $R_{1}(t-1), \quad R_{2}(t), \quad R_{2}(t-1), \quad R_{3}(t), \quad R_{3}(t-1)$, $\left.R_{4}(t), R_{4}(t-1)\right)$

Model 3: $Q(t+1)=f(Q(t), Q(t-1), Q(t-2)$, $R_{1}(t), \quad R_{1}(t-1), \quad R_{1}(t-2), \quad R_{2}(t), \quad R_{2}(t-1)$, $R_{2}(t-2), \quad R_{3}(t), \quad R_{3}(t-1), \quad R_{3}(t-2), \quad R_{4}(t)$, $\left.R_{4}(t-1), R_{4}(t-2)\right)$ 

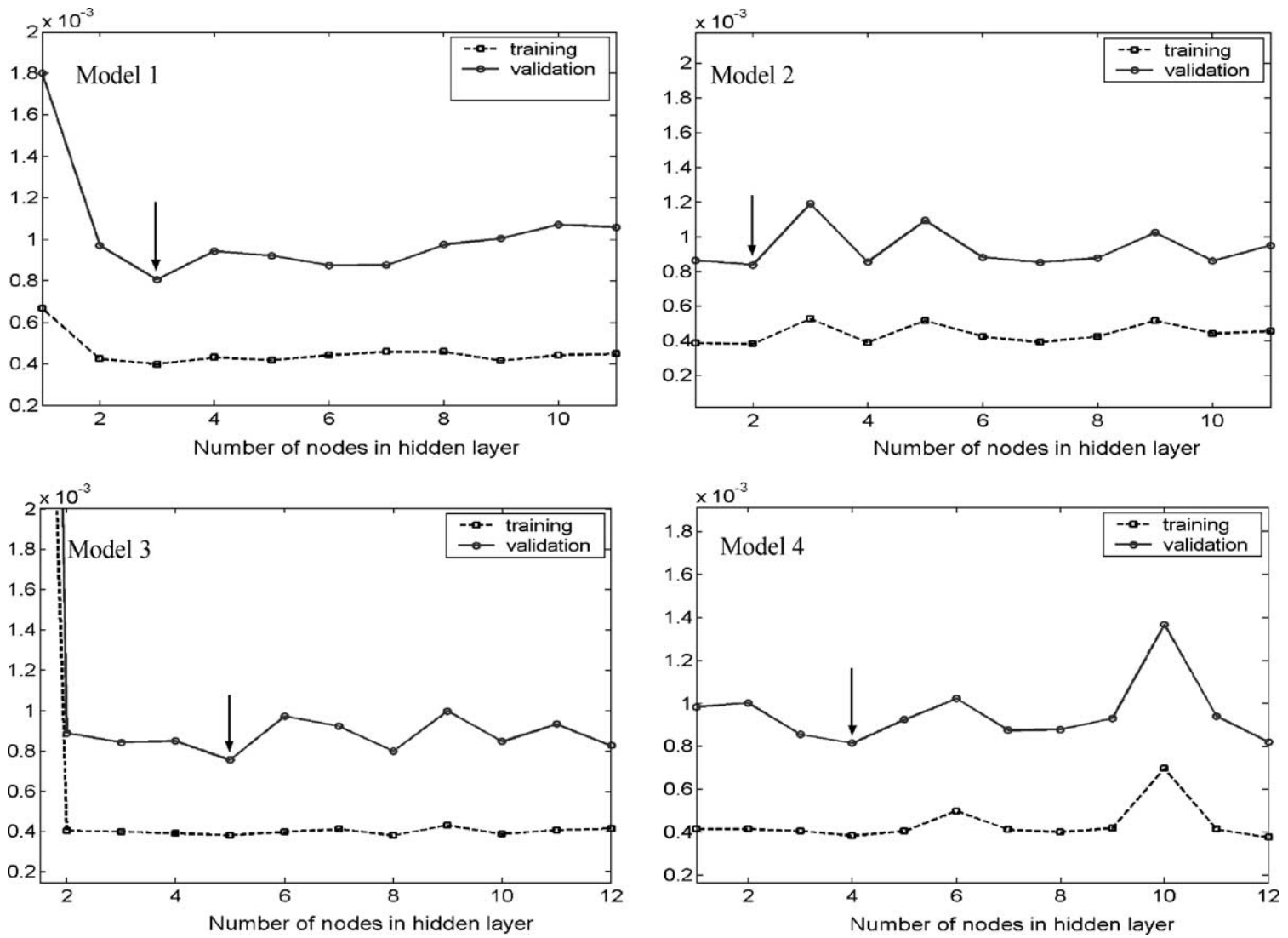

Fig. 6. The selection of hidden neurons from models 1 to 4 for CG.

Model 4: $Q(t+1)=f(Q(t), Q(t-1), Q(t-2)$, $Q(t-3), R_{1}(t), R_{1}(t-1), R_{1}(t-2), R_{1}(t-3)$, $R_{2}(t), \quad R_{2}(t-1), \quad R_{2}(t-2), \quad R_{2}(t-3), \quad R_{3}(t)$, $R_{3}(t-1), R_{3}(t-2), R_{3}(t-3), R_{4}(t), R_{4}(t-1)$, $\left.R_{4}(t-2), R_{4}(t-3)\right)$

where $Q(t-i)$ represents the value of the Lan-Yang Bridge streamflow gauge station at time $t-i$, and $R_{1}(t-i), \quad R_{2}(t-i), \quad R_{3}(t-i)$ and $R_{4}(t-i)$ represents the precipitation of the four rainfall gauge stations at time $t-i$. Because the drainage area of the study watershed is less than $1000 \mathrm{~km}^{2}$ and the basin slope is quite steep, the time of concentration is short. Consequently, the input pattern is focused on the previous one to $3 \mathrm{~h}$ information only. Also, because of the feedback quality of recurrent neural networks, their input dimensions are values of each gauge station at the present time $t$; this is a particular characteristic as far as the dynamicfeedback neural network is concerned.

\subsubsection{Selection of the number of hidden neurons}

The determination of the appropriate number of hidden neurons is very important for the success of the ANNs. If the hidden layer has too many neurons this may cause each hidden neuron to memorize one of the input patterns, and thereby reduce the generalization capabilities and increase the training time but without significant improvement in training results (Ranjithan et al., 1993). Imrie et al. (2000) indicate that too few hidden neurons may have insufficient degrees of freedom to capture the underlying relationships in the data. Since, there is not a systematic or standard way to 
Table 2

Comparison of different standard BP architectures

\begin{tabular}{llrllll}
\hline & \multirow{2}{*}{ Architecture } & \multicolumn{2}{l}{ MAE } & & \multicolumn{2}{l}{ RMSE } \\
\cline { 3 - 4 } \cline { 6 - 7 } \cline { 6 - 7 } & & Training & Validation & & Training & Validation \\
\hline Model 1 & $5-11-1$ & 85 & 93 & & 195 & 209 \\
Model 2 & $10-11-1$ & 106 & 107 & & 243 & 212 \\
Model 3 & $15-11-1$ & 89 & 90 & & 197 & 171 \\
Model 4 & $20-11-1$ & 107 & 119 & & 252 & 292 \\
\hline
\end{tabular}

decide on the number of neurons in the hidden layer, the best way to select the hidden neurons is by trial-and-error.

\subsection{Selecting the best static-feedforward neural network structure}

First, we use case 1 with four different input models to determine the standard $\mathrm{BP}$ and $\mathrm{CG}$ network structures by using trial-and-error. Figs. 5 and 6 show the selection of the hidden neurons from model 1 to model 4 for standard BP and CG, respectively. The hidden neurons from 1 to 15 are varied in each model and all the simulations are terminated after 10,000 iterations. Tables 2 and 3 show the comparison of different models for standard BP and CG, respectively, and the performance of different models is presented based on the criteria of mean absolute error (MAE) and root mean square error (RMSE) as shown below:

MAE $=\frac{\sum_{t=1}^{N}\left|\left(Q_{\text {sim }}(t)-Q_{\text {obs }}(t)\right)\right|}{N}$

Table 3

Comparison of different CG architectures

\begin{tabular}{lllllll}
\hline & \multirow{2}{*}{ Architecture } & \multicolumn{2}{l}{ MAE } & & \multicolumn{2}{l}{ RMSE } \\
\cline { 6 - 6 } \cline { 6 - 6 } & & Training & Validation & & Training & Validation \\
\hline Model 1 & $5-3-1$ & 49 & 52 & 110 & 115 \\
Model 2 & $10-2-1$ & 48 & 53 & & 103 & 103 \\
Model 3 & $15-5-1$ & 45 & 48 & & 97 & 105 \\
Model 4 & $20-4-1$ & 53 & 53 & & 113 & 120 \\
\hline
\end{tabular}

$\mathrm{RMSE}=\sqrt{\frac{\sum_{t=1}^{N}\left(Q_{\mathrm{sim}}(t)-Q_{\mathrm{obs}}(t)\right)^{2}}{N}}$

where $Q_{\text {sim }}$ is the forecasted value, $Q_{\text {obs }}$ is the observed value, and $N$ is the number of data points.

The results show that Model 3 produces the best performance for both the standard $\mathrm{BP}$ and $\mathrm{CG}$ network structures. This result indirectly provides evidence that the average lag time is no more than $3 \mathrm{~h}$. Comparing the results in Table 2 and 3, it is easy to tell that the $\mathrm{CG}$ method is superior to the standard BP method in all the models and phases. Consequently, we only use the CG algorithm for training the static-feedforward neural network and compare with RTRL.

\section{Results and discussion}

The input dimensions of RTRL are five which are established by using the current four rain-gauges and the streamflow data, while the processing layer is constructed to have five neurons. To compare the model accuracy of CG and RTRL, the performances of these two methods are evaluated based on the criteria of MAE, RMSE, relative mean absolute error (RMAE) and $\hat{Q}_{\mathrm{p}}$. The RMAE and $\hat{Q}_{\mathrm{p}}$ are defined as follows

$\mathrm{RMAE}=\frac{\mathrm{MAE}}{\bar{Q}}$

Table 4

Results of the CG and RTRL for case 1

\begin{tabular}{lllllr}
\hline Case 1 & $Q_{\mathrm{p}}$ & $\hat{Q}_{\mathrm{p}}$ & MAE & RMAE & RMSE \\
\hline $\begin{array}{l}\text { Training } \\
\quad\end{array}$ & & & & & \\
$\quad$ CG & 3530 & 2501 & 45 & 0.078 & 97 \\
$\quad$ RTRL & & 2700 & 52 & 0.090 & 110 \\
Validation & & & & & \\
$\quad$ CG & 3350 & 3294 & 48 & 0.093 & 105 \\
RTRL & & 3220 & 59 & 0.114 & 149 \\
Testing & & & & & \\
$\quad$ CG & 3640 & 2790 & 59 & 0.078 & 118 \\
RTRL & & 3339 & 83 & 0.110 & 166 \\
\hline
\end{tabular}




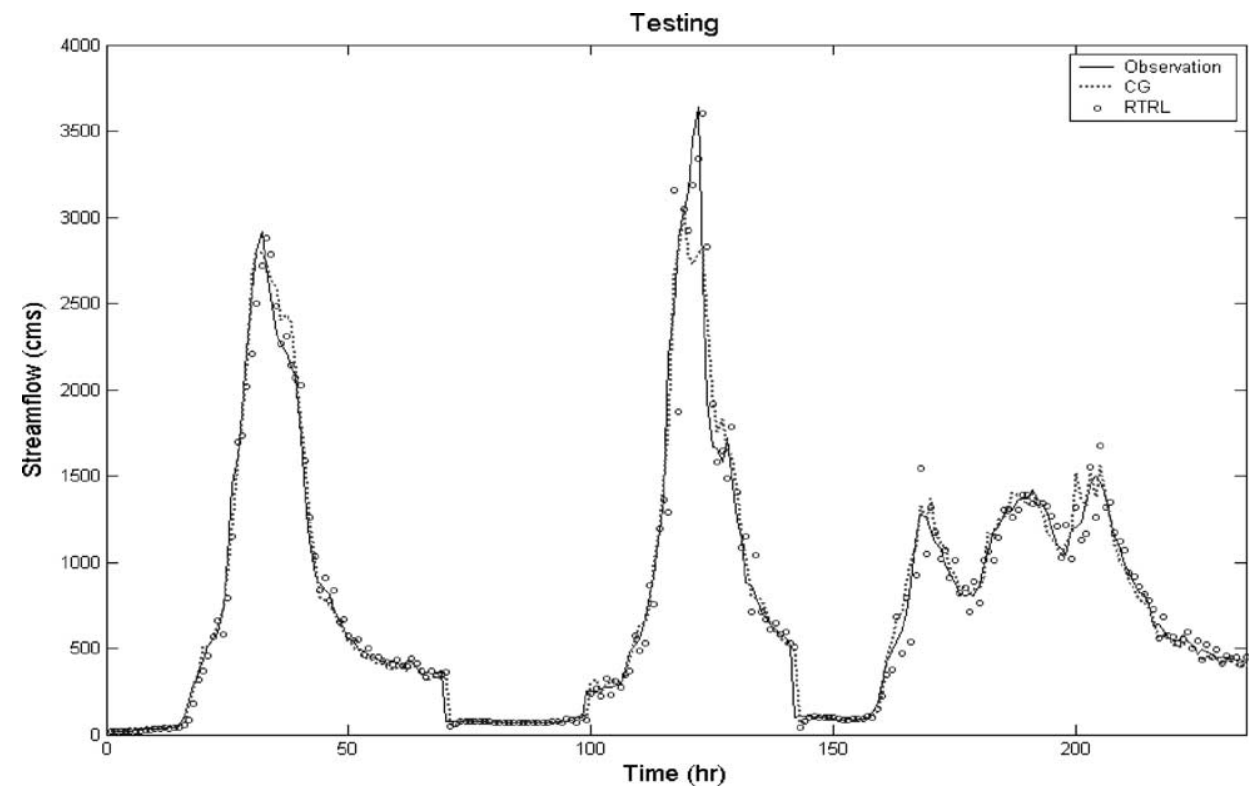

Fig. 7. Observed versus forecasted streamflow for a testing period of case 1.
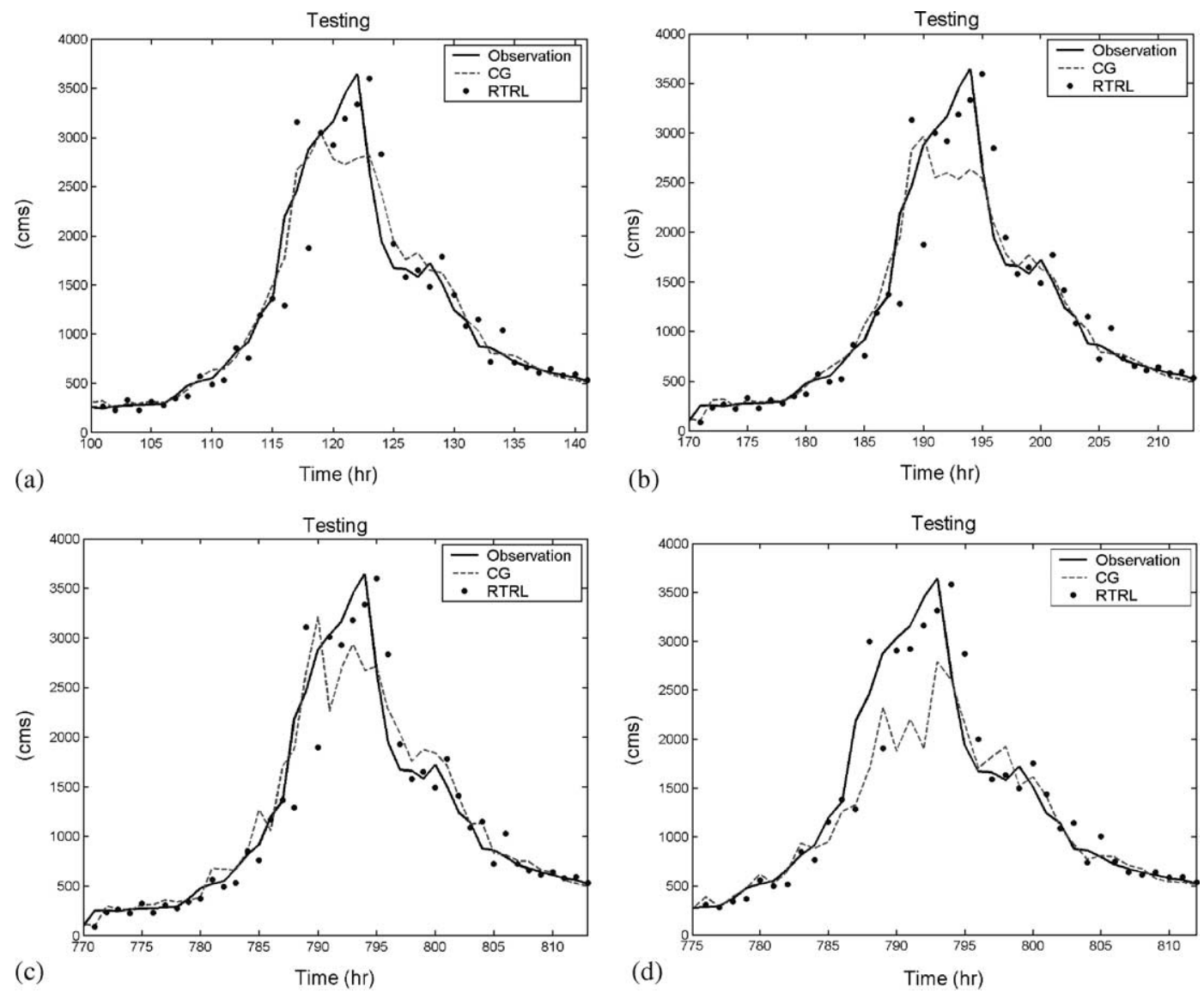

Fig. 8. Observed versus forecasted streamflow in the vicinity of peak flow (a) case 1, (b) case 2, (c) case 3 and (d) case 4. 
Table 5

Results of the CG and RTRL for case 2

\begin{tabular}{llllll}
\hline Case 2 & $Q_{\mathrm{p}}$ & $\hat{Q}_{\mathrm{p}}$ & MAE & RMAE & RMSE \\
\hline $\begin{array}{l}\text { Training } \\
\quad\end{array}$ & & & & & \\
$\quad$ CG & 3090 & 2986 & 43 & 0.075 & 79 \\
$\quad$ RTRL & & 2892 & 49 & 0.086 & 96 \\
Validation & & & & & \\
CG & 3350 & 3300 & 51 & 0.099 & 104 \\
RTRL & & 3181 & 58 & 0.112 & 129 \\
Testing & & & & & \\
$\quad$ CG & 3640 & 2633 & 111 & 0.135 & 225 \\
RTRL & & 3329 & 102 & 0.124 & 225 \\
\hline
\end{tabular}

$\hat{Q}_{\mathrm{p}}$ is the estimated value at the time that the observed peak flow occurred (cms).

Table 4 summarizes the comparative results of the CG and RTRL methods for case 1. It appears that both methods produce good forecasting of the streamflow in all phases. We can also find that the CG method obtains better performance (in terms of small MAE, RMAE, and RMSE) than the RTRL, because of sufficient training length and content in case 1 . However, the $\hat{Q}_{\mathrm{p}}$ values produced by the RTRL method are superior to CG. Fig. 7 shows a comparison of observed versus forecasted hourly streamflow for a testing period of case 1. Fig. 8(a) is the enlarged peak flow of case 1, so we can easily distinguish between the two models' performance.

The results of both methods for case $2-4$ are presented in Tables 5-7. Fig. 8(b-d) show the observed versus forecasted streamflow by $\mathrm{CG}$ and

Table 6

Results of the CG and RTRL for case 3

\begin{tabular}{llllll}
\hline Case 3 & $Q_{\mathrm{p}}$ & $\hat{Q}_{\mathrm{p}}$ & MAE & RMAE & RMSE \\
\hline $\begin{array}{l}\text { Training } \\
\quad\end{array}$ & & & & & \\
$\quad$ CG & 3530 & 2474 & 55 & 0.098 & 128 \\
$\quad$ RTRL & & 2735 & 57 & 0.102 & 129 \\
Validation & & & & & \\
CG & 3020 & 2861 & 47 & 0.093 & 88 \\
RTRL & & 2741 & 37 & 0.074 & 79 \\
Testing & & & & & \\
$\quad$ CG & 3640 & 2667 & 81 & 0.126 & 165 \\
RTRL & & 3337 & 68 & 0.105 & 143 \\
\hline
\end{tabular}

Table 7

Results of the CG and RTRL for case 4

\begin{tabular}{llllll}
\hline Case 4 & $Q_{\mathrm{p}}$ & $\hat{Q}_{\mathrm{p}}$ & MAE & RMAE & RMSE \\
\hline Training & & & & & \\
$\quad$ CG & 2916 & 2725 & 40 & 0.082 & 70 \\
$\quad$ RTRL & & 2818 & 47 & 0.097 & 84 \\
Validation & & & & & \\
CG & 3020 & 2739 & 54 & 0.107 & 112 \\
RTRL & & 2755 & 37 & 0.074 & 78 \\
Testing & & & & & \\
$\quad$ CG & 3640 & 2790 & 113 & 0.168 & 249 \\
RTRL & & 3315 & 74 & 0.110 & 158 \\
\hline
\end{tabular}

RTRL in the vicinity of peak flow in cases $2-4$, respectively. In the training stage, the CG method has slightly better performance than the RTRL in all three cases; however, the RTRL method produces much better performance than the CG method in terms of smaller MAE, RMAE, RMSE and high $\hat{Q}_{\mathrm{p}}$ value in testing phases of all cases. The results demonstrate that RTRL is relatively stable and performs well in all cases, whereas CG presents satisfying performance in the testing phase only when the training data has enough length and sufficient contents.

Moreover, another important observation that can be made from the static-feedforward neural network forecasted result is with reference to the selection of the training data set. Fig. 9 depicts the scatter plot of CG of all cases. Fig. 9(a) shows the reasonable and consistent performance in all three phases in case 1 . Fig. 9(b) shows the model performance for case 2 which includes the same training length of data sets as case 1 , but no extreme events are included. It appears the accuracy in the testing phase was inferior to case 1 even though a more accurate result is obtained in the training phase. Fig. 9(d) represents a good spread and the results in the training set of case 4 are the closest to the ideal line, which only has a short length of data and is without extreme events. However, in the testing phase, it has the widest spread and departure from the ideal line results. That is, it has the worst performance. Examining the forecasted results of the CG method for cases 1-4, it can be seen that the CG method produces much less accurate results in the test phase than the training phase for all the cases except case 1. These results 
(a)

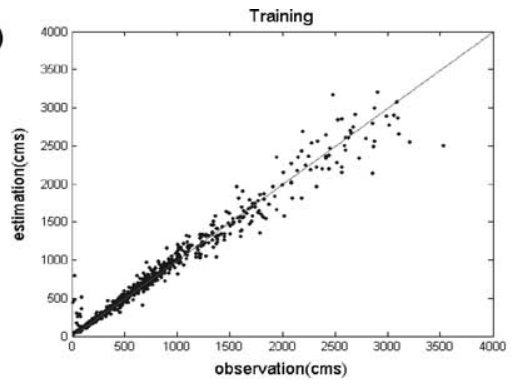

(b)

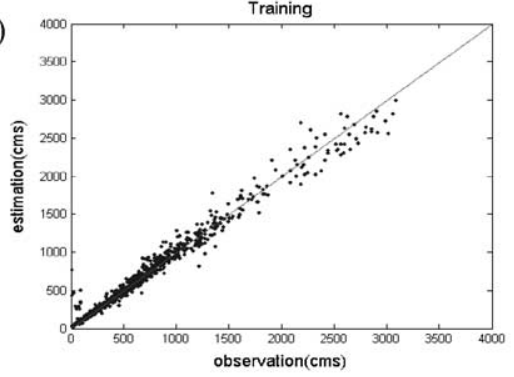

(c)

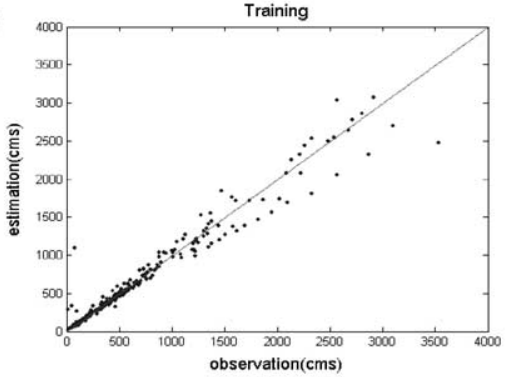

(d)

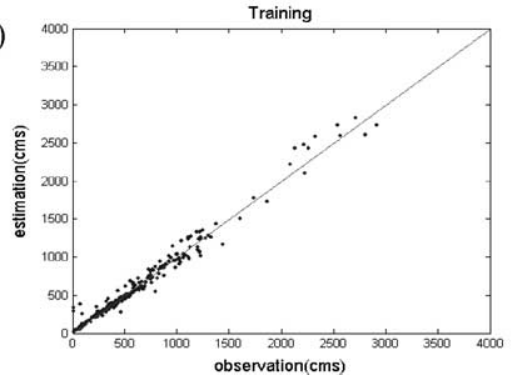

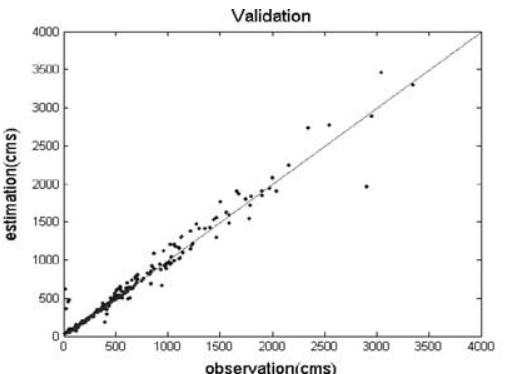
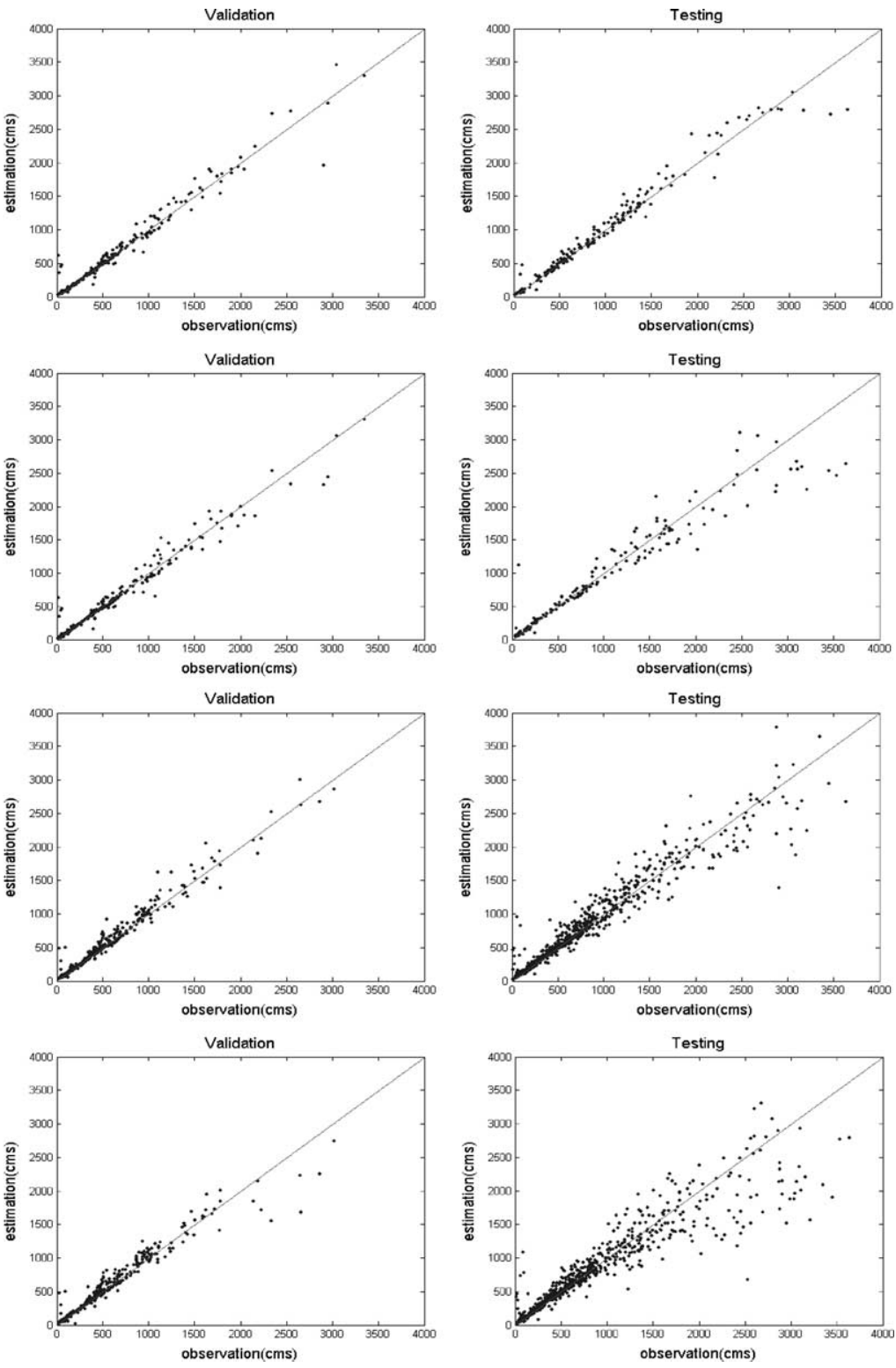

Fig. 9. Comparison of observed and forecasted streamflow of CG (a) case 1, (b) case 2, (c) case 3 and (d) case 4.

might indicate that the static-feedforward neural network with the CG algorithm could not produce satisfactory results when there is not a sufficient and adequate training data set to build a suitable inputoutput relationship.

Fig. 10 depicts the scatter plot of RTRL for all cases. Because of the continually updating and learning characteristic of the RTRL network, the operation is regarded as a whole procedure for each case, different from the CG network that divides available data into three phases. Fig. 10(a-d) shows that the performance of RTRL of all four cases is quite stable and consistent. It appears that the RTRL produces acceptable results insensitive to the length and contents of the data set. Moreover, the RTRL has the ability to capture the main trend or characteristics 

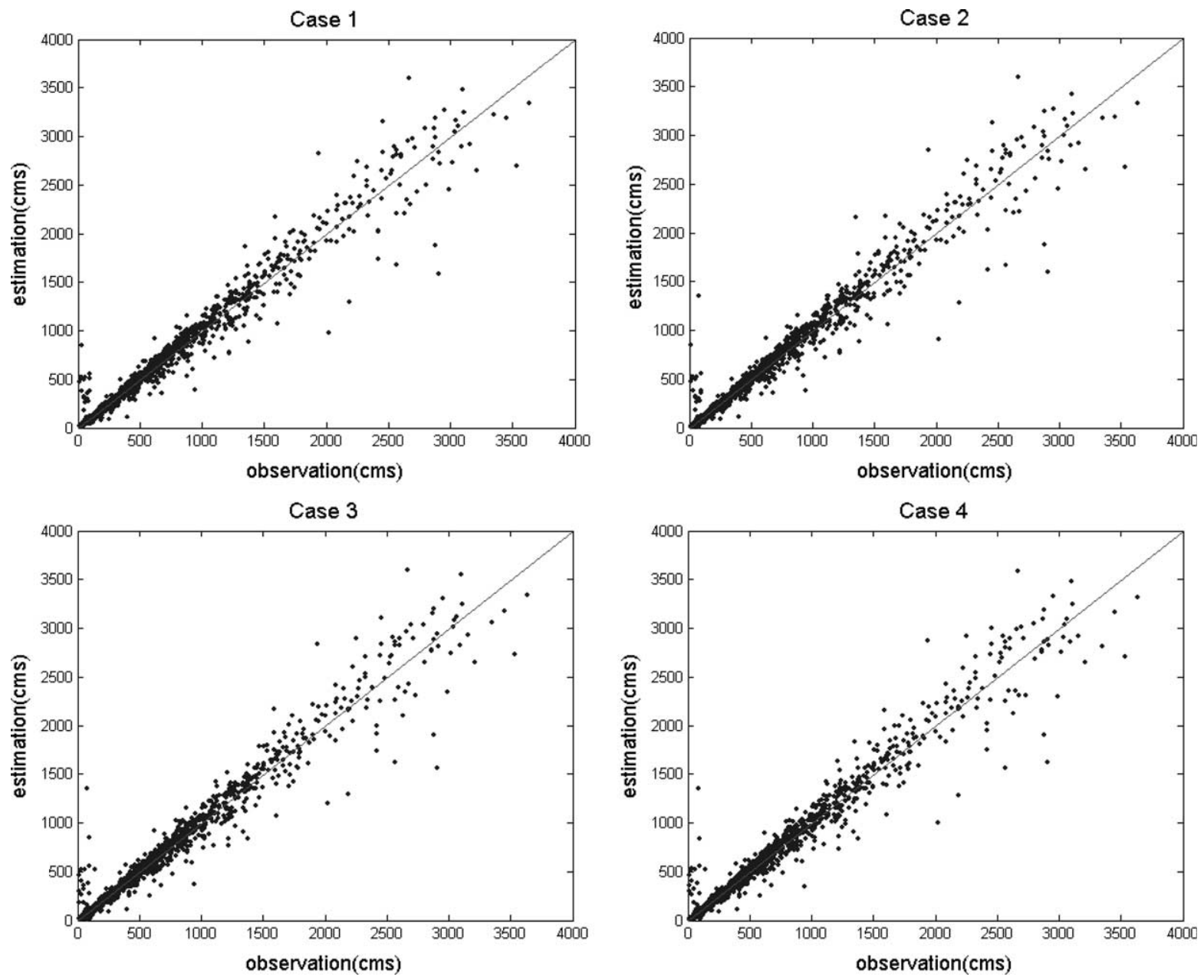

Fig. 10. Comparison of observed and forecasted streamflow of RTRL for all cases.

of the time series. These results demonstrate that the dynamic-feedback neural network with the RTRL algorithm has high practicability and accurate ability for streamflow forecasting.

\section{Conclusions}

Various types of neural networks have been used to construct the rainfall-runoff models and led to satisfactory results. To learn the stability and effectiveness of two primary types of neural networks, i.e. static-feedforward and dynamic-feedback, trained through different length and content of data, we design four different input patterns and four cases of data sets of the Lan-Yang River in Taiwan to train, validate, and test the networks. The standard BP and CG algorithms are used to search the optimizing values of connecting weights of the static-feedforward network, and the RTRL algorithm is used for a dynamic-feedback network.

In comparing the searching algorithms for a staticfeedforward network, the results show that the CG method is superior to the standard BP method in terms of efficiency and effectiveness of the constructed network's performance. For a comparison of the static neural network using the CG algorithm with the dynamic neural network using RTRL, the following results are obtained. First, both static and dynamic neural networks yield a reasonably good forecast if 
there is adequate length and content of data included (case 1). Even though, the static-feedforward network produces slightly better performance (in terms of small MAE and RMSE values) than the dynamic network, we notice that the dynamic network better captures the peak flows. Second, in cases 2-4, for which non-sufficient length or content of data is involved in the training phase, the results show that the dynamic network has significantly better performance than the static network, especially in the testing phase. The static network produces poor performance (in terms of larger MAE and RMSE, and highly underestimates the peak flow) in the testing phase. These results suggest that the static-feedforward neural network with the $\mathrm{CG}$ algorithm could produce satisfactory results only when there is a sufficient and adequate training data set. Third, the feedforward neural networks executed fixed-weight mapping from the input space to the output space. Due to fixed weights, the output is solely determined by the present input state to the network and not the initial and past states of the neurons in the network. Consequently, the input-output mapping of the feedforward neural networks is static and the networks would be trained to store the information content in the training data and could only produce the memory structures within processes. On the contrary, the feedback neural networks do allow initial and past state involvement along with serial processing. These networks provide a representation of dynamic internal feedback loops to store information for later use, to enhance the efficiency of learning, and to carry out the sequential temporal behavior. Furthermore, the RTRL algorithm helps to continually update the feedback neural network for learning, and this feature is especially important and useful for grasping the extraordinary time-varying characteristics of rainfall-runoff processes.

\section{Acknowledgements}

This paper is based on partial work supported by National Science Council, ROC (Grant No. NSC912313-B-002-343). In addition, the authors are indebted to the reviewers for their valuable comments and suggestions.

\section{References}

Abrahart, R.J., See, L., 2000. Comparing neural network and autoregressive moving average techniques for the provision of continuous river flow forecasts in two contrasting catchments. Hydrological Processes 14, 2157-2172.

ASCE Task Committee on Application of Artificial Neural Networks in Hydrology, 2000a. Artificial neural networks in hydrology I: preliminary concepts. Journal of Hydrologic Engineering 5 (2), 115-123.

ASCE Task Committee on Application of Artificial Neural Networks in Hydrology, 2000b. Artificial neural networks in hydrology II: Hydrologic applications. Journal of Hydrologic Engineering 5 (2), 124-137.

Beven, K., 2001. How far can we go in distributed hydrological modeling. Hydrology and Earth System Science 5, 1-12.

Cameron, D., Kneale, P., See, L., 2002. An evaluation of traditional and a neural net modeling approach to flood forecasting for an upland catchment. Hydrological Processes 16, 1033-1046.

Campolo, M., Andreussi, P., Soldati, A., 1999. River flood forecasting with a neural network model. Water Resources Research 35 (4), 1191-1197.

Chang, L.C., Chang, F.J., 2001. Intelligent control for modeling of real time reservoir operation. Hydrological Processes 15, $1621-1634$.

Chang, F.J., Chen, Y.C., 2001. A counterpropagation fuzzy-neural network modeling approach to real time stream flow prediction. Journal of Hydrology 245, 153-164.

Chang, F.J., Chen, Y.C., 2003. Estuary water-stage forecasting by using Radial Basis Function neural network. Journal of Hydrology 270, 158-166.

Chang, F.J., Liang, J.M., Chen, Y.C., 2001. Flood forecasting using radial basis function neural network. IEEE Transactions on Systems, Man and Cybernetics Part C: Applications and Reviews 31 (4), 530-535.

Chang, F.J., Chang, L.C., Huang, H.L., 2002. Real-time recurrent neural network for stream-flow forecasting. Hydrological Processes 16, 2577-2588.

Chang, L.C., Chang, F.J., Chiang, Y.M., 2004. A two-step ahead recurrent neural network for streamflow forecasting. Hydrological Processes 18, 81-92.

Coulibaly, P., Anctil, F., Bobee, B., 2001. Multivariate reservoir inflow forecasting using temporal neural networks. Journal of Hydrologic Engineering 6 (5), 367-376.

Dawson, C.W., Wilby, R.L., 1998. An artificial neural network approach to rainfall-runoff modeling. Hydrological Sciences 43 (1), 47-67.

Dawson, C.W., Wilby, R.L., 2001. Hydrological modeling using artificial neural networks. Progress in Physical Geography 25 (1), 80-108.

Elshorbagy, A., Simonovic, S.P., Panu, U.S., 2000. Performance evaluation of artificial neural networks for runoff prediction. Journal of Hydrologic Engineering ASCE 5 (4), 424-427.

French, M.N., Krajewski, W.F., Cuykendall, R.R., 1992. Rainfall forecasting in space and time using a neural network. Journal of Hydrology 137, 1-31. 
Golob, R., Stokelj, T., Grgic, D., 1998. Neural-network-based water inflow forecasting. Control Engineering Practice 6, 593-600.

Govindaraju, R.S., Ruo, A.R., 2000. Artificial Neural Networks in Hydrology. Kluwer Academic Publishers, Dordrecht.

Ham, F.M., Kostanic, I., 2001. Principles of neurocomputing for science and engineering. McGraw-Hill, New York.

Haykin, S., 1999. Neural Network: a Comprehensive Foundation, second ed., Prentice Hall, Englewood, Cliffs, NJ.

Hopfield, J.J., 1982. Neural networks and physical systems with emergent collective computational abilities. Proceedings of the National Academy of Sciences of the United States of America 79 (8), 2254-2258.

Hsu, K.L., Gupta, H.V., Sorooshian, S., 1995. Artificial neural network modeling of the rainfall-runoff process. Water Resources Research 31 (10), 2517-2530.

Hydrological Engineering Center, 1990. HEC-1 Flood Hydrograph Package, Program Users Manual. US Army Crops of Engineers: Davis, C.A.,.

Imrie, C.E., Durucan, S., Korre, A., 2000. River flow prediction using artificial neural network: generalisation beyond the calibration range. Journal of Hydrology 233, 138-153.

Karunanithi, N., Grenney, W.J., Whitley, D., 1994. Neural network for river flow prediction. Journal of Computing in Civil Engineering 8, 201-220.

Kohonen, T., 1982. Self-organized formation of topologically correct feature maps. Biological Cybernetics 43, 59-69.

Luk, K.C., Ball, J.E., Sharma, A., 2000. A study of optimal model lag and spatial inputs to artificial neural network for rainfall forecasting. Journal of Hydrology 227, 56-65.

Maier, H.R., Dandy, G.C., 2000. Neural networks for the prediction and forecasting of water resources variables: a review of modelling issues and applications. Environmental Modelling and Software 15 (1), 101-124.

McCulloch, W.S., Pitts, W., 1943. A logical calculus of the ideas immanent in nervous activity. Bulletin of Mathematical Biophysics 5, 115-133.

Minns, A.W., Hall, M.J., 1996. Artificial neural networks as rainfall-runoff models. Hydrological Sciences 41 (3), 399-417.
Powell, M.J.D., 1985. Radial basis functions for multivariable interpolation: a review, IMA Conference on Algorithms for the Approximation of Functions and Data, RMCS, Shrivenham, England, pp. 143-167.

Ranjithan, S., Eheart, J.W., Garrett, J.H. Jr., 1993. Neural networkbased screening for groundwater reclamation under uncerntainty. Water Resources Research 29 (3), 563-574.

Rumelhart, D.E., Hinton, G.E., Williams, R.J., 1986. Learning internal representations by error propagation. Parallel Distributed Processing 1, 318-362.

Sajikumar, N., Thandaveswara, B.S., 1999. A non-linear rainfallrunoff model using an artificial neural network. Journal of Hydrology 216, 32-55.

Salas, J.D., Tabios, G.Q., Bartolini, P., 1985. Approaches to multivariate modeling of water resources time series. Water Resources Bulletin 21 (4), 683-708.

Shamseldin, A.Y., 1997. Application of a neural network technique to rainfall-runoff modeling. Journal of Hydrology 199, $272-294$.

Sivakumar, B., Jayawardena, A.W., Fernando, T.M.K.G., 2002. River flow forecasting-use of phase-space reconstruction and artificial neural networks approaches. Journal of Hydrology 265 , $225-245$.

Smith, J., Eli, R.N., 1995. Neural network models of rainfall-runoff process. Journal of Water Resources Planning and Management 121 (6), 499-508.

Thirumalaiah, K., Deo, M.C., 1998. River stage forecasting using artificial neural network. Journal of Hydrologic Engineering 3 (1), 26-32.

Tokar, A.S., Johnson, P.A., 1999. Rainfall-runoff modeling using artificial neural networks. Journal of Hydrologic Engineering 4 (3), 232-239.

Williams, R., Zipser, D., 1989. A learning algorithm for continually running fully recurrent neural network. Neural Computation 1 , 270-280.

Zealand, C.M., Burn, D.H., Simonovic, S.P., 1999. Short term stream flow forecasting using artificial neural networks. Journal of Hydrology 214, 32-48. 